\title{
Mean Expression Value Normalized and Absolute Quantified miR-21 Found to be a Potential Diagnostic and Prognostic Marker for IgA Nephropathy
}

\author{
Santosh Kumar ${ }^{1}$, C. Priscilla ${ }^{1}$, Sreejith Parameswaran², Deepak Gopal \\ Shewade ${ }^{3}$, A. Charan Goud ${ }^{3}$, Rajesh Nachiappa Ganesh ${ }^{1, *}$ \\ 'Department of Pathology, Jawaharlal Institute of Postgraduate Medical Education \\ and Research, Puducherry 605006, India \\ 2Department of Nephrology, Jawaharlal Institute of Postgraduate Medical Education \\ and Research, Puducherry 605006, India \\ ${ }^{3}$ Department of Pharmacology, Jawaharlal Institute of Postgraduate Medical \\ Education and Research, Puducherry 605006, India
}

Article Type: Article

Article Citation: Santosh Kumar, Priscilla C, Sreejith Parameswaran, Deepak Gopal Shewade, Charan Goud A, Rajesh Nachiappa Ganesh. Mean expression value normalized and absolute quantified miR-21 found to be a potential diagnostic and prognostic marker for IgA nephropathy. Indian Journal of Science and Technology. 2020; 13(09), 1078-1088.D01:10.17485/ijst/2020/ v013i09/149977

Received date: February 3, 2020

Accepted date: February 17, 2020

*Author for correspondence: Rajesh Nachiappa Ganesh drngrajesh@gmail.com P Department of Pathology, Jawaharlal Institute of Postgraduate Medical Education and Research, Puducherry 605006, India

\section{Abstract}

Background/objectives: miR-21 has been linked to renal fibrosis and miR-155 too has shown different expression in IgAN whose pathogenesis is partially understood. In this study, we explored the utility of miR-21 and miR-155 in IgA nephropathy. Methods: We recruited $40 \mathrm{lg} A$ nephropathy (IgAN) patients and 15 healthy controls (HC). $2 \mathrm{ml}$ blood sample was collected from each study participant. Mean expressed value normalization and absolute and relative quantification methods were used for quantitative polymerase chain reaction (PCR) experiment for miRNA expression. Logistic regression analysis was performed for diagnostic and prognostic value of miR-21. Findings: Mean expressed value normalization reduced the variation in groups and between groups without changing the mean value. miR-155 showed similar expression in IgAN and healthy controls. There was three-fold increases in miR-21 in IgAN than the HC. miR-21 was able to differentiate the mesangial hypercellularity and tubular atrophy/interstitial fibrosis stages with sensitivity 84 and 70 percent, respectively. Applications: miR-155 can be used as a reference control gene for IgAN on quantitative PCR-based experiments. miR-21 can be used as a diagnostic and prognostic marker for lgAN.

Keywords: miR-21, miR-155, IgAN, Tubular Atrophy, Interstitial Fibrosis. 


\section{Introduction}

microRNAs (miRNAs) are small RNA molecules of 18 to 22 nucleotides in length which play an important role in protein formation by interacting with mRNAs post transcriptionally [1]. It is assumed that multiple cellular interactions take place during miRNA biogenesis which may help to understand the disease pathology [2] Furthermore, one miRNA can interact with multiple mRNA and vice versa and hence the presence, absence, increased, or decreased level of miRNA in any patient sample could be a sign of specific disease condition [3]. Sequencing, microarray, and polymerase chain reaction (PCR) are major techniques to identify and/or measure the miRNAs in fluid and tissue samples. Out of all the methods, PCR is considered as the gold standard method for introductory as well as confirmatory method for the miRNA expression [4]. There are multiple stages involved in real-time PCR-based miRNA expression. It starts with sample (fluid or/and tissue) collection, miRNA isolation, complementary DNA (cDNA) conversion, and amplification stage. These multistage procedure behind miRNA expression cause technical variations which result into false positive. Normalization techniques are used in real-time PCRbased miRNA expression experiments to reduce the technical variations for better and accurate measurement of biological variations of the disease [5] Mean expression value is frequently used for the normalization process of miRNA expression based studies [6]. This method is used to find the best suitable endogenous reference control for miRNA expression. Technical variation can be reduced at the post RNA isolation stage by using an equal concentration of miRNA for cDNA conversion for each sample. But, the miRNA yield in isolated RNA sample use to be very low in quantity and hence it is very difficult to use this method. An exogenous miRNA is used as internal control to see the technical variation in RNA isolation process. There is different opinion to use exogenous miRNA as internal control in normalization process $[7,8]$.

Relative quantification method is used for miRNA expression based on real-time PCR experiment [9]. Absolute quantification of miRNA is rarely reported in publication by citing its lesser value than the relative quantification of miRNA. The logarithmic transform of critical values (Ct) PCR amplification result of miRNA is used for comparing miRNA values with other clinical (including pathology) and biochemical parameters of the disease. The clinical and biochemical parameters are expressed in the form of arithmetic values where Ct value in PCR is exponential. Logarithmic transform of Ct does not represent the PCR results in absolute and correct form to compare it with clinical, pathological, and biochemical parameters. It results in false positive.

IgA nephropathy (IgAN) is a slow progressive autoimmune disease [10]. Young adults are at higher risk (we reported the trend in our population in the previous study). The diagnosis of this disease is completely dependent on kidney biopsy [11]. Kidney biopsy is an invasive and complicated procedure and it is not performed at preliminary level and is neither repeated frequently neither repeated frequently. This practice causes a late diagnosis of IgAN.

In this study, we have used mean expression method to normalize the miRNA experiment on real-time PCR method and used absolute quantification method which is the exact liner transform of the exponential Ct values to see the association of miR-21 with 
histopathological and biochemical parameters in IgAN patients. miR-21 is also known as fibromiR which is associated with the renal fibrosis while miR-155 is associated with inflammatory regulatory cells in IgAN [12-19] whose prognosis is highly.

\section{Materials and Methods}

\subsection{Patient Selection}

We recruited 40 IgAN patients and 15 healthy persons as study participants after taking their informed written consent following the inclusion and exclusion criteria. The participants were in the age group of 15-70 years. The consecutive native biopsies, reported as primary IgA nephropathy, were included in the study after excluding secondary causes of IgA nephropathy, patients with systemic lupus erythematosus (SLE), Henoch Schonlein purpura (HSP), other autoimmune disease, renal carcinoma, human immunodeficiency virus infection, hepatitis, diabetes mellitus, and infection-related glomerulonephritis. Institute human ethics committee approval was taken for the study.

\subsection{Sample Collection}

Two ml blood was collected in EDTA tube after taking the informed written consent from the study participants. The sample was processed within one hour of collection. Plasma was centrifuged at $3000 \mathrm{~g}$ value for five minutes at four degree temperature. The supernatants were stored immediately in aliquots at -80 degree Celsius till further processing.

\section{3. miRNA Quantification}

miRNA expression was performed at two different times. The first experiment was performed on 30 study samples. Fifteen samples were taken from each of the IgAN and healthy control (HC) groups based on age and sex-matched criteria. This experiment was performed to see the miRNA (miR-21 and miR-155) expression level differences between the diseased and healthy groups. The second experiment was carried on 40 IgAN patients. This experiment was performed to see the association of miRNA (miR-21) level with the histopathological and biochemical parameters in IgAN patients.

A synthetic miRNA $(5 \mu \mathrm{l})$, as an exogenous internal control, was spiked into $200 \mu \mathrm{l}$ plasma as the first step followed by adding the lysis buffer for the RNA isolation process. Silica bead spin column-based total RNA isolation method was performed. The isolated RNA was collected into $20 \mu \mathrm{l}$ of nuclease-free water. The quality of RNA was checked by Qubit 3.0 (ThermoFisher Scientific) fluorometer instrument. miRNA, DNA, and protein quantity were checked by Qubit 3 instrument using specific miRNA, DNA, and protein kits (Invitrogen), respectively. Only that isolated RNA was further processed where protein and DNA were not traceable. cDNA conversion was done by Reverse Transcriptase (RT) enzyme. $8 \mu$ of RNA and $2 \mu \mathrm{l}$ of RT enzyme were used as input along with cDNA mix for cDNA conversion. cDNA conversion was performed at PCR thermal setup of 
$25^{\circ} \mathrm{C}$ for $15 \mathrm{~min}, 42{ }^{\circ} \mathrm{C}$ for $60 \mathrm{~min}$, and $85^{\circ} \mathrm{C}$ for $5 \mathrm{~min}$. The cDNA was immediately used as continuous process for further amplification of target miRNAs and exogenous internal control miRNA. Two $\mu$ of cDNA was used for each amplification reaction. The specific primers and probes were used for miR-21, miR-155, and exogenous miRNA. The necessary reagents were procured by Helini biomolecules, India. The ABI QS3 (Thermo Fisher Scientific) real-time PCR instrument was used for cDNA conversion and miRNA amplification and quantification. Amplification was done in two steps. The first step was used as Taq enzyme activation stage at $95^{\circ} \mathrm{C}$ for $15 \mathrm{~min}$. The second step was run in 45 cycles at $95{ }^{\circ} \mathrm{C}$ for $20 \mathrm{~s}$ as denaturation stage, $56^{\circ} \mathrm{C}$ for $20 \mathrm{~s}$ as annealing stage, and $72{ }^{\circ} \mathrm{C}$ for $20 \mathrm{~s}$ as extension. The miRNA analysis was done in triplicate and the mean value was used for analysis. A standard curve was prepared in serial dilution with known concentration (copy number) of miRNA to quantify the miRNAs by absolute expression method. Absolute quantification was done for the copy number calculation of miR-21 [20-23] Relative gene expression based on $\mathbf{2}^{\wedge-\Delta \Delta C t}$ method was used for the fold change calculation [24-26].

\subsection{Histopathological Analysis}

Native kidney biopsies of IgAN patients were analyzed and reported by light microscopy and immunofluorescence. MEST-C (M-Mesangial hypercellularity, E-Endocapillary hypercellularity, S-Segmental glomerulosclerosis, T-Tubular atrophy/Interstitial fibrosis, C-Crescents) scoring under Oxford classification was used and biopsy findings were documented [27-29].

\subsection{Normalization Process of Real-Time Quantitative PCR Data}

\subsubsection{Experiment 1}

Ct value of exogenous miRNA was subtracted from the target miRNA in each sample. Deviation from mean was calculated and further added to the target miRNA Ct value. The following steps were followed: Step 1. Target_miRNA_Ct - Exogenous_miRNA_Ct = del_Ct; Step 2. del_Ct - mean_del_Ct $=d e v \_C t$; Step 3. Target_miRNA_Ct $+d e v \_C t=$ Nomalized_Target_miRNA_Ct. All the three steps were performed for each sample in all the groups (IgAN and HC) of each target miRNA (miR-21 and mIR-155). The normalized Ct value (Nomalized_Target_miRNA_Ct) was used for the gene expression analysis.

\subsubsection{Experiment 2}

miR-21 concentration (copy number/ $\mu$ l eluted RNA) was calculated based on the standard curve method. Step 1 and Step 2 of Experiment 1 were followed to get the deviation $\mathrm{Ct}$ value (dev_Ct) for miR-21. Normalized miR-21 copy number (per $\mu$ l eluted RNA) was calculated based on the equation: $C_{n}=C_{i}(1+e)^{\text {dev }}-C_{t}$; where, $C_{n}=$ normalized miR21 copy number, $C_{i}=$ initial miR-21 copy number, $\mathrm{e}=$ efficiency of PCR amplification, dev_C $\mathrm{t}=$ deviation in $\mathrm{Ct}$ between the target and exogenous miRNA. 


\subsection{Primers and Probe}

The forward and reverse primers used for miR-21 were CAACACCAGTCGATGGG, GCGATCGGTAAGTACCTGA, respectively. The forward and reverse primers used for miR-155 were CAACACCAGTCGATGGG, GCGATCGGTAAGTACCTGA, respectively. The probe used in the experiment was CGTACGTACGTA. All the nucleotide sequences are in five to three prime directions.

\subsection{Statistical Analysis}

Shapiro-Wilk normality test performed to check the data distribution. T-test and Wilcoxon's rank sum test was performed for probability distinction. F-test performed to calculate the differences within and between groups. Logistic regression method used for the prognostic prediction of miRNAs against MEST-C score. Receiver operating characteristic (ROC) curve was made by pROC package of $\mathrm{R}$ [30]. $p$ Value less than 0.05 was considered as significant. Copy number value was rounded off to the nearest integer. All the statistical analysis performed using $\mathrm{R}$ version 3.6.2 [31].

\subsection{Ethical Approval}

"All procedures performed in this study involving human participants were in accordance with the ethical standards of the institute human ethics committee and with the 1964 Helsinki Declaration and its later amendments or comparable ethical standards." The study has been started after the approval of institute human ethics committee (JIP/IEC/ SC/2015/19/785). Informed written consent was taken from all individual participants included in the study.

\section{Results}

The mean age of IgAN patients $(n=40)$ was 29.15 years with standard deviation (SD) 9.03. The male and female IgAN patients were at 52.50 and 47.50 percent, respectively. The median serum creatinine value was $1.65(\mathrm{mg} / \mathrm{dl})$ with an inter quartile range $(1.2-3.45)$ $\mathrm{mg} / \mathrm{dl}$. The mean systolic and diastolic blood pressure were $123.80(\mathrm{SD}=18.70)$ and 81.50 $(\mathrm{SD}=11.56) \mathrm{mmHg}$, respectively.

\subsection{Experiment 1}

The mean Ct, SD, and variance in group (Var) for miR-155 in IgAN patients were 29.383, 0.196 , and 0.038 , respectively. The mean Ct, SD, and Var for miR-155 in healthy controls were 29.388, 0.089, and 0.088, respectively. After normalization with exogenous miRNA, the mean Ct, SD, and Var for miR-155 in IgAN patients were 29.385, 0.501, and 0.251, respectively. The mean Ct, SD, and Var for miR-155 in healthy controls after normalization were $29.388,0.494$, and 0.244 , respectively. Normalization with exogenous miRNA 
reduced the variation in the group without changing the mean $\mathrm{Ct}$ values of the groups. The difference in mean Ct value between diseased (IgAN) and healthy groups before and after normalization was 0.004 and 0.002 , respectively. The ratio of variances of $\mathrm{Ct}$ (miR-155) values between the diseased and healthy groups, before and after normalization were 4.818 and 1.031. Normalization with exogenous miRNA again reduced the variation between the diseased and healthy groups. These results establish miR-155 as an endogenous reference

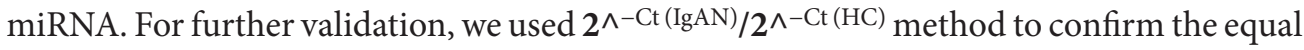
expression of miR-155 in IgAN and healthy conditions. The ratio was found to be 1.0018 which confirmed and validated the miR-155 usage as endogenous reference miRNA in IgAN patients.

The mean Ct, SD, and Var for miR-21 in IgAN patients before normalization were $20.171,0.849$, and 0.721 , respectively. The mean Ct, SD, and Var for miR-21 in healthy controls before normalization were $21.748,0.894$, and 0.799 , respectively. The mean Ct, SD, and Var for miR-21 in IgAN after normalization were 20.176, 1.283, and 1.648, respectively. The mean Ct, SD, and Var for miR-21 in healthy controls after normalization were $21.746,1.326$, and 1.758 , respectively.

The difference in mean $\mathrm{Ct}$ values between the diseased and healthy groups for miR-21 was 1.577 and $p$ value was found to be 0.008 . miR-155 used as an endogenous reference control gene to calculate the miR-21 expression in IgAN patients based on $2^{\wedge-\Delta \Delta C t}$ method. miR-21 was found to be 2.963 fold increased in IgAN patients in comparison to healthy controls.

\subsection{Experiment 2}

miR-21 was able to differentiate the mesangial hypercellularity and tubular atrophy/ interstitial fibrosis stages in IgAN patients. The median copy number and inter quantile range (IQR) of mesangial proliferation stages $\mathrm{M} 0$ and $\mathrm{M} 1$ are 56 (11-83) and 122 (77-288) respectively with $p$ value 0.032 . The median copy number and IQR of tubular atrophy/ interstitial fibrosis stages T0 and T1 were 176 (81-297) and 56 (29-103) respectively with $p$ value 0.005 . ROC curve based on logistic regression analysis confirmed the predictive value of miR-21 for the mesangial proliferation and tubular atrophy/interstitial fibrosis stages in IgAN patients (Figures 1 and 2). The area under curve (AUC) of ROC for mesangial hypercellularity $(\mathrm{M})$ and tubular atrophy/interstitial fibrosis $(\mathrm{T})$ stages were 0.74 and 0.79 , respectively. The sensitivity (SE), specificity (SP), and confidence interval (CI) for the statistical models (Figures 1 (a) and 2(a)) for $\mathrm{M}$ and $\mathrm{T}$ were $0.84,0.87,0.52-$ 0.97 (DeLong's method) and 0.70, 0.80, 0.64-0.94 (DeLong's method), respectively. The cutoff value of miR-21 copy number for $\mathrm{M}$ and $\mathrm{T}$ stages was 58 and 68, respectively.

\section{Discussion}

There is a diverse opinion on reference gene selection for PCR-based miRNA gene expression studies $[7,32,33]$. Different normalization methods are in use for the selection of reference gene for miRNA expression studies $[5,34]$. We used mean expression value 


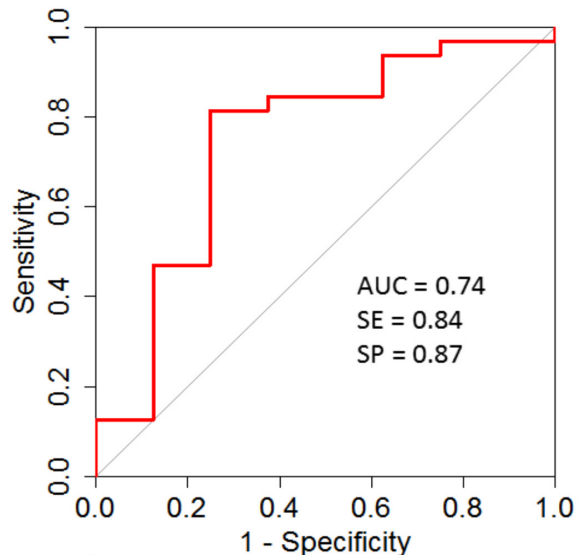

(a)

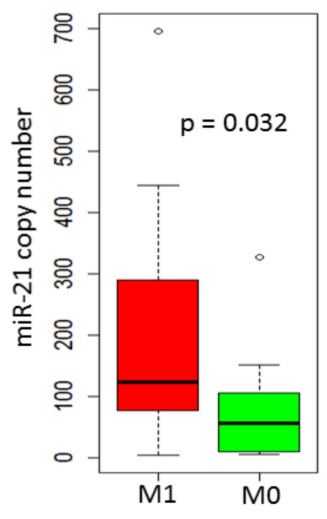

(b)

(a): AUC - Area under receiver operating characteristic curve, SE - sensitivity, SP -specificity

(b): miR-21 copy number is expressed in per $\mu$ l eluted RNA, M1 - mesangial score $>0.5$, MO - mesangial score $<0.5$

FIGURE 1. Prognostic value of miR-21 for mesangial hypercellularity in $\lg A$ nephropathy.

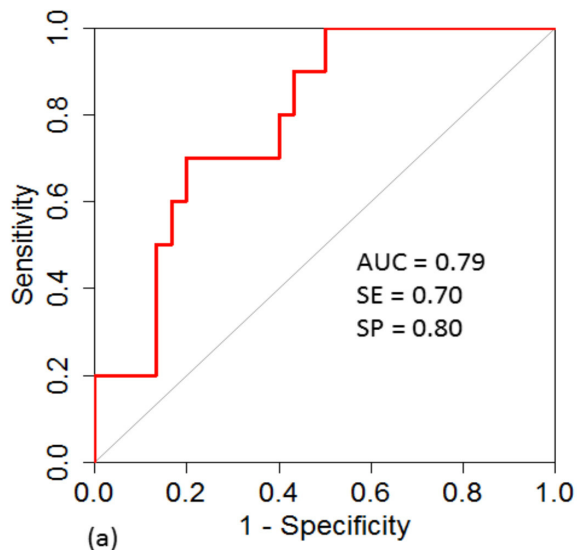

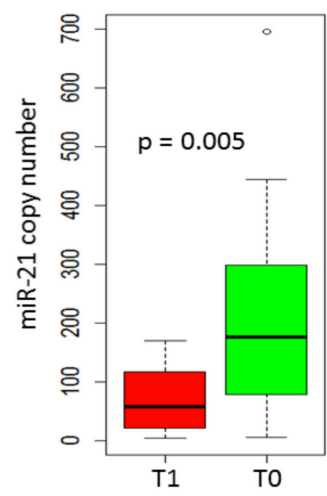

(b)

(a): AUC - Area under receiver operating characteristic curve, SE - Sensitivity, SP - specificity

(b): miR-21 copy number is expressed in per $\mu$ l eluted RNA, T1 - tubular atrophy/interstitial fibrosis ( T2 is included) $>25 \%$, T0 - Tubular atrophy/interstitial fibrosis $\leq 25 \%$

FIGURE 2. Prognostic value of miR-21 for tubular atrophy/interstitial fibrosis in IgA nephropathy.

method in our experiment. This method reduced the group variance without changing the mean values. Previous studies have shown miR-155 expression level increased or decreased in urine, serum, and kidney tissue samples in IgAN, lupus nephritis, and other kidney disease cases $[14,17,18,35]$. We are the first one to report miR-155 as a potential 
reference gene in IgA nephropathy in plasma samples for quantitative PCR-based gene expression study. We used miR-155 as reference gene and studied miR-21 through relative and absolute experimentation. miR-21 was found overexpressed in IgAN cases than the $\mathrm{HC}$ and here we do not have any conflict with previous studies carried on IgAN.

The PCR amplification is an exponential process and it should not be compared with linear form of data produced in histopathological and biochemical experiments. We used a copy number of miR-21 to see its potential as a prognostic tool to differentiate the different stages of disease defined by histopathological and biochemical parameters. The copy number is calculated by transforming the $\mathrm{Ct}$ values, which is exponential by nature, into linear arithmetic values.

miR-21 has been associated with renal fibrosis [36-38]. It was also found that miR-21 increased with the disease (IgAN) severity in previous studies carried on serum, urine, kidney tissues and it was able to differentiate the segmental sclerosis (S) stages [39-41]. Our result partly verified the previous studies. We, too, found miR-21 level elevated in IgAN. We could not find miR-21 differentiating the S score in IgAN. Further, we found that miR-21 was able to differentiate the mesangial proliferation and tubular atrophy/ interstitial fibrosis $(\mathrm{T})$ stages in IgAN. We got a contradictory result in our experiment. miR-21 level was higher in T0 stage than T1 stage. Out of all the 40 IgAN patients, T2 score was found only in four patients and, so, we included T2 score in T1 score category. $\mathrm{T}$ score is considered an independent strong prognostic parameter of MEST-C Oxford histological score of IgAN. Increased level of each element in MEST-C score indicates the severity of IgAN. But, miR-21 level was increased in M1 and decreased in T1 conditions. All the M0T1 score IgAN patients have reduced miR-21 level as well as in M1T1 than the M0T0 and M1T0 conditions. It was observed that T1 superseded M1 and brought the miR-21 level down. It is an unusual result and first time seen in IgAN cases. We could not find any significant correlation between miR-21 and serum creatinine or with any other histopathological or biochemical parameters. The cutoff value of miR-21 copy number for $\mathrm{M}$ and $\mathrm{T}$ stage differentiation is 58 and 68 and in opposite direction to each other. So, we can use 58 and 68 copy number of miR-21 as a range to confirm IgAN condition. The lower and upper values to confirmatory range of miR-21 can be used for the prognosis of the disease. There is a need to study miR-21 in IgAN patients at different population level to know the exact role of miR-21 in IgAN.

\section{Conclusion}

miR-155 can be used as an internal reference gene for gene expression studies on IgAN. Mean expression value reduces the variation within and between groups without changing the mean value. Copy number of miRNAs should be used for statistical analysis purpose when miRNA is compared with clinical, pathological, or biochemical parameters. miR-21 has potential to be used as diagnostic and prognostic marker for IgAN.

\section{Conflict of Interest}

None declared. 


\section{Acknowledgement}

This work was supported by Science and Engineering Research Board (SERB), Government of India (File number - EMR/2016/003382) and Jawaharlal Institute of Postgraduate Medical Education and Research, Puducherry, India.

\section{References}

1. Bartel DP. MicroRNAs: Genomics, biogenesis, mechanism, and function. Cell. 2004; 116(2), 281-297. DOI: 10.1016/S0092-8674(04)00045-5.

2. Treiber T, Treiber N, Meister G. Regulation of microRNA biogenesis and its crosstalk with other cellular pathways. Nature Reviews Molecular Cell Biology. 2019, 20(1), 5-20. DOI:10.1038/ s41580-018-0059-1.

3. Trionfini P, Benigni A, Remuzzi G. MicroRNAs in kidney physiology and disease. Nature Reviews Nephrology. 2015, 11(1), 23-33. DOI: 10.1038/nrneph.2014.202.

4. Benes V, Castoldi M. Expression profiling of microRNA using real-time quantitative PCR, how to use it and what is available. Methods. 2010, 50(4), 244-249. DOI: 10.1016/j.ymeth.2010.01.026.

5. Andersen CL, Jensen JL, Ørntoft TF. Normalization of real-time quantitative reverse transcription-PCR data: A model-based variance estimation approach to identify genes suited for normalization, applied to bladder and colon cancer data sets. Cancer Research. 2004, 64(15), 5245-5250. DOI: 10.1158/0008-5472.CAN-04-0496.

6. Mestdagh P, Van Vlierberghe P, De Weer A. A novel and universal method for microRNA RT-qPCR data normalization. Genome Biology. 2009, 10(6). DOI: 10.1186/gb-2009-10-6-r64.

7. Derveaux S, Vandesompele J, Hellemans J. How to do successful gene expression analysis using real-time PCR. Methods. 2010, 50(4), 227-230. DOI: 10.1016/j.ymeth.2009.11.001.

8. Vandesompele J, De Preter K, Pattyn F. Accurate normalization of real-time quantitative RT-PCR data by geometric averaging of multiple internal control genes. Genome Biology. 2002, 3(7). http://www.ncbi.nlm.nih.gov/pubmed/12184808

9. Kroh EM, Parkin RK, Mitchell PS, Tewari M. Analysis of circulating microRNA biomarkers in plasma and serum using quantitative reverse transcription-PCR (qRT-PCR). Methods. 2010, 50(4), 298-301. DOI: 10.1016/j.ymeth.2010.01.032.

10. Zhu X, Li H, Liu Y. IgA Nephropathy. Seminars in Nephrology. 2017, 38(5), 1-8. DOI: 10.1016/j. semnephrol.2018.05.016.

11. Rodrigues JC, Haas M, Reich HN. IgA nephropathy. Clinical Journal of the American Society of Nephrology. 2017, 12(4), 677-686. DOI: 10.2215/CJN.07420716.

12. Hennino MF, Buob D, Van Der Hauwaert C. MiR-21-5p renal expression is associated with fibrosis and renal survival in patients with IgA nephropathy. Science Reports. 2016, 6, 1-9. DOI: $10.1038 /$ srep27209.

13. Wang G, Kwan BCH, Lai FMM, Chow KM, Li PKT, Szeto CC. Urinary miR-21, miR-29, and miR-93: novel biomarkers of fibrosis. American Journal of Nephrology. 2012. DOI: $10.1159 / 000343452$.

14. Khoshmirsafa M, Kianmehr N, Falak R. Elevated expression of miR-21 and miR-155 in peripheral blood mononuclear cells as potential biomarkers for lupus nephritis. International Journal of Rheumatic Diseases. 2019, 22(3), 458-467. DOI: 10.1111/1756-185X.13410.

15. Chau BN, Xin C, Hartner J. MicroRNA-21 promotes fibrosis of the kidney by silencing metabolic pathways. Science Translational Medicine. 2012, 4(121). DOI: 10.1126/scitranslmed.3003205. 
16. Loboda A, Sobczak M, Jozkowicz A, Dulak J. TGF- $\beta$ 1/Smads and miR-21 in renal fibrosis and inflammation. Mediators of Inflammation. 2016, 2016, 8319283. DOI: 10.1155/2016/8319283.

17. Wang G, Kwan BCH, Lai FMM, Chow KM, Li PKT, Szeto CC. Elevated levels of miR-146a and miR-155 in kidney biopsy and urine from patients with IgA nephropathy. Disease Markers. 2011, 30(4), 171-179. DOI: 10.3233/DMA-2011-0766.

18. Hu YY, Dong W Da, Xu YF. Elevated levels of mir-155 in blood and urine from patients with nephrolithiasis. BioMed Research International. 2014, 2014, 295651. DOI: 10.1155/2014/295651.

19. Yang L, Zhang X, Peng W, Wei M, Qin W. MicroRNA-155-induced T lymphocyte subgroup drifting in IgA nephropathy. International Urology and Nephrology. 2017, 49(2), 353-361. DOI: 10.1007/s11255-016-1444-3.

20. Bustin SA. Absolute quantification of MRNA using real-time reverse transcription polymerase chain reaction assays. Journal of Molecular Endocrinology. 2000, 25(2), 169-193. DOI: 10.1677/ jme.0.0250169.

21. Whelan JA, Russell NB, Whelan MA. A method for the absolute quantification of cDNA using real-time PCR. Journal of Immunological Methods. 2003, 278(1-2), 261-269. DOI: 10.1016/ S0022-1759(03)00223-0.

22. Ferracin M, Lupini L, Salamon I. Absolute quantification of cell-free microRNAs in cancer patients. Oncotarget. 2015, 6(16), 14545-14555. DOI: 10.18632/oncotarget.3859.

23. Larionov A, Krause A, Miller WR. A standard curve based method for relative real time PCR data processing. BMC Bioinformatics. 2005, 6(62). DOI: 10.1186/1471-2105-6-62.

24. Livak KJ, Schmittgen TD. Analysis of relative gene expression data using real-time quantitative PCR and the 2- $\Delta \Delta$ CT method. Methods. 2001, 25(4), 402-408. DOI: 10.1006/meth.2001.1262.

25. Schmittgen TD, Livak KJ. Analyzing real-time PCR data by the comparative C(T) method. Nature Protocols. 2008, 3(6), 1101-1108. http://www.ncbi.nlm.nih.gov/pubmed/18546601

26. Pfaffl MW. A new mathematical model for relative quantification in real-time RT-PCR. Nucleic Acids Research. 2001, 29(9), e45. http://www.ncbi.nlm.nih.gov/pubmed/11328886

27. Barbour SJ, Espino-Hernandez G, Reich HN. The MEST score provides earlier risk prediction in lgA nephropathy. Kidney International. 2016, 89(1), 167-175. DOI: 10.1038/ki.2015.322.

28. Barbour S, Reich H. An update on predicting renal progression in IgA nephropathy. Current Opinion in Nephrology and Hypertension. 2018, 27(3), 214-220. DOI: 10.1097/ MNH.0000000000000405.

29. Trimarchi H, Barratt J, Cattran DC, et al. Oxford classification of IgA nephropathy 2016: an update from the IgA nephropathy classification working group. Kidney International. 2017, 91(5), 1014-1021. DOI: 10.1016/j.kint.2017.02.003.

30. Robin X, Turck N, Hainard A. pROC: an open-source package for R and S+ to analyze and compare ROC curves. BMC Bioinformatics. 2011, 12(1), 77. DOI: 10.1186/1471-2105-12-77.

31. R: a language and environment for statistical computing. R Foundation for Statistical Computing: Vienna, Austria. https://www.R-project.org/. Date accessed: 12/12/2019.

32. Stolf-Moreira R, Lemos EG de M, Abdelnoor RV. Identification of reference genes for expression analysis by real-time quantitative PCR in drought-stressed soybean. Pesqui Agropecuária Bras. 2011, 46(1), 58-65. DOI: 10.1590/s0100-204x2011000100008.

33. Bae IS, Seo KS, Kim SH. Identification of endogenous microRNA references in porcine serum for quantitative real-time PCR normalization. Molecular Biology Reports. 2018, 45(5), 943-949. DOI: $10.1007 / \mathrm{s} 11033-018-4242-4$.

34. Udvardi MK, Czechowski T, Scheible W-R. Eleven golden rules of quantitative RT-PCR. Plant Cell Online. 2008, 20(7), 1736-1737. DOI: 10.1105/tpc.108.061143.

35. Szeto CC, Wang G, Ng JKC. Urinary miRNA profile for the diagnosis of IgA nephropathy. BMC Nephrology. 2019, 20(1), 77. DOI: 10.1186/s12882-019-1267-4. 
36. Sole, Moline, Vidal, Ordi-Ros, Cortés-Hernández. An exosomal urinary miRNA signature for early diagnosis of renal fibrosis in lupus nephritis. Cells. 2019, 8(8), 773. DOI: 10.3390/ cells 8080773 .

37. Liu L, Wang Y, Yan R. BMP-7 inhibits renal fibrosis in diabetic nephropathy via miR-21 downregulation. Life Science. 2019, 238, 116957. DOI: 10.1016/j.lfs.2019.116957.

38. Nakhjavani M, Etemadi J, Pourlak T, Mirhosaini Z, Vahed SZ, Abediazar S. Plasma levels of miR-21, miR-150, miR-423 in patients with lupus nephritis. Iranian Journal of Kidney Diseases. 2019, 13(3), 198-206. http://www.ijkd.org/index.php/ijkd/article/view/4310

39. Wu J, Zhang H, Wang W. Plasma microRNA signature of patients with IgA nephropathy. Gene. 2018, 649, 80-86. DOI: 10.1016/j.gene.2018.01.050.

40. Selvaskandan H, Pawluczyk I, Barratt J. MicroRNAs: a new avenue to understand, investigate and treat immunoglobulin a nephropathy? Clinical Kidney Journal. 2018, 11(1), 29-37. DOI: 10.1093/ckj/sfx096.

41. Liang S, Cai GY, Duan ZY. Urinary sediment miRNAs reflect tubulointerstitial damage and therapeutic response in IgA nephropathy. BMC Nephrology. 2017, 18(1), 1-9. DOI: 10.1186/ s12882-017-0482-0. 